\title{
Filtering intrusion detection alarms Using Ant Clustering approach
}

\author{
Salah Ghodhbani ${ }^{1}$, Farah Jemili ${ }^{2}$ \\ Student, Department of Computer Engineering, Sousse University, ISG, Tunisia ${ }^{1}$ \\ Assistant Professor, Department of Computer Engineering, Sousse University, ISITCOM, Tunisia ${ }^{2}$
}

\begin{abstract}
With the growth of cyber attacks, information safety has become an important issue all over the world. Many firms rely on security technologies such as intrusion detection systems (IDSs) to manage information technology security risks. IDSs are considered to be the last line of defense to secure a network and play a very important role in detecting large number of attacks. However the main problem with today's most popular commercial IDSs is generating high volume of alerts and huge number of false positives. This drawback has become the main motivation for many research papers in IDS area. Hence, in this paper we present a data mining technique to assist network administrators to analyze and reduce false positive alarms that are produced by an IDS and increase detection accuracy. Our data mining technique is unsupervised clustering method based on hybrid ANT algorithm. This algorithm discovers clusters of intruders' behavior without prior knowledge of a possible number of classes, then we apply K-means algorithm to improve the convergence of the ANT clustering. Experimental results on real dataset show that our proposed approach is efficient with high detection rate and low false alarm rate.
\end{abstract}

Keywords: Intrusion detection system, Alarm filtering, ANTClass, ant clustering, intruders' behaviors, false alarms

\section{INTRODUCTION}

With the explosive increase in number of services accessible through the Internet, information security needs to be carefully concerned and a sufficient protection is needed against cyber attacks. Intrusion detection systems (IDSs) are an essential component in computer network security. It monitors target sources of activities, collects and inspects audit data looking for evidences of intrusive behaviors. When it detects suspicious or malicious attempts, an alarm is raised giving the network administrator the opportunity to react promptly. IDS can be classified as Host-based Intrusion Detection System (HIDS) which protects a certain host or system, and Network-based Intrusion detection system (NIDS), which protects a network of hosts and systems [2]. The NIDS uses the audit data; an audit trail is a record of actions on a system that are logged to a file in chronologically sorted order. The alarms generated by a NIDS inform network administrators that their network is possibly under attack [1]. However, NIDS might generate huge amount of alarms during the detection stage, which exhausts system administrator by rendering a large amount of a false alarms. So we need more intelligent attack analysis to deal with false alarms problems and increase detection accuracy.

Various techniques have been proposed to analyze alarms and reduce false positives. Ning and $\mathrm{Xu}$ [3] proposed a technique that automatically learn attack strategies as acyclic graphs and extract them from correlated intrusion alarms. Kruegel et al [4] performed real-time verification of attacks in which the NIDS reduces the priority of non real attacks and, thus, differentiates between false and nonrelevant alarms. Rachman [5] positioned a data
48/IJARCCE.2014.31246 
mining layer, to represent the baseline of security combining ANT and K-mean algorithms. Basically, system deployment and to analyze new data against ANT preprocessed the data to produce a number this baseline, clusters with centers of intruders' behaviors. On the other hand, K-mean algorithm is applied to refine the final results of ANT module to get a more uniform as an analysis layer within NIDS. Julisch and Dacier partition of clusters.

[6] handled alarms by identifying root causes and We empirically show, using real world data that our removing them. Cuppens et al. [7] analyzed alarms proposed technique uncovers attack vectors hiding in by implementing functions to manage, cluster, merge normal clusters. That is, it reduces false negatives and correlate alarms. produced by the NIDS and it competes well with Faour et al. [8] proposed Self-Organizing Maps other data mining techniques like SOM and GSOM. (SOM) coupled with Bayesian networks for alarm This paper is organized as follows. Section 2 filtering. But, their SOM work resulted in fixed provides a background on ant based clustering and network architecture in terms of number and map ANTClass algorithm. Section 3 explains the units arrangement. . In [1] a data mining technique architecture of ANTClass method for alarms filtering. based on Growing Hierarchical Self-Organizing Map Section 4 presents and discusses our empirical (GHSOM) which adjusts its topology during the results. The last section offers the conclusions.

learning process according to the inputs data (alarms) to reduce false positive alarms and to

\section{BACKGROUND ON ANT CLUSTERING}

assist system administrators in analyzing alarms An easy way to comply with the conference paper generated by the IDS. The proposed algorithm aims formatting requirements is to use this document as a template and simply type your text into it.

to explore the hidden structure of alarm data, and to A. Ant based clustering

uncover false alarms (FP \& FN) hiding in normal Ant colonies provide a means to formulate some clusters. Considerably, a data sample consisting of powerful nature-inspired heuristics for solving the 1849 data patterns including 6 web attack scenarios clustering problems. Several clustering methods based were tested using the proposed approach. The on ant behavior have been proposed in the results show that the proposed algorithm outperforms literature. Ant-based clustering sorting was first SOM algorithm in terms of both false positives and introduced by Deneubourg et al. [10] He proposed false negatives which reduced from $15 \%$ to $4.7 \%$ the basic ant model for clustering. He focused on and from $16 \%$ to $4 \%$ respectively. A major limitation clustering objects by using a group of real-world in the previous solutions of alert correlation is that robots. In his model, the ants would walk randomly the methods used led to increase false positives. on the workspace, picking or dropping one data In this work, we propose a system based on element from it. The ants possessed only local ANTClass [10] algorithm, for the first time, as Ant perceptual capabilities. They could sense the clustering approach for the problem of alarm filtering. surrounding objects were similar or not to the object, Our approach, presents an unsupervised learning they were carrying. Based on this information, they method that does not assume prior knowledge of the would perform the pick or drop action. structure of the data, which supports administrators to explore alarms pattern in order to analyze intruders' behaviors and to uncover false positive and false negative alarms. The proposed method provides better detection and lower false positive rate by
The basic model (BM) of Deneubourg can be described as follows: The data items are randomly scattered into a two-dimensional grid. Initially, each data object that represents a multi-dimensional 
pattern is randomly distributed over the 2D space.

Each ant moves randomly around this grid picking IF $f\left(O_{i}\right) \geq k_{2} \mathrm{~s}$

and dropping the data items. The decision to pick up or drop an item is random but is influenced by the data items in the ant's immediate neighborhood. The probability of dropping an item is increased if ants are surrounded with similar data in the neighborhood. In contrast, the probability of picking an item is increased if a data item is surrounded by dissimilar data, or when there is no data in its

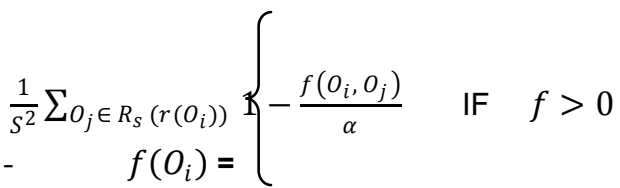

(3)

0 Else neighborhood. In this way, clustering of the elements on the 2D grid is obtained.

Where:

Lumer and Faieta [11] modified Deneubourg et al.'s BM [10] using a dissimilarity-based evaluation of the local density, in order to make it suitable for data clustering and it has subsequently been used in data mining [12]. This algorithm is called LF model or • $\mathrm{d}(\mathrm{oi}, \mathrm{oj})$ is the dissimilarity between pair of standard ant clustering algorithm (SACA). In this objects (oi, oj) algorithm, each ant-like agent cannot communicate with each other, and they can only sense the • similarity of the objects in their immediate region. dissimilarity

Lumer and Faieta [11] have introduced the notion of short-term memory within each agent. Each ant •

$\mathrm{k} 1$ and $\mathrm{k} 2$ are two constants that play a remembers a small number of locations where it has role similar to $k 1$ and $k 2$ in the BM. successfully dropped an item. And so, when picking a new item this memory is consulted in order to bias the direction in which the ant will move. Thus, the ant tends to move towards the location it last dropped a similar item. Lumer and Faieta [11] define picking up $(\mathrm{Pp})$ and dropping probabilities $(\mathrm{Pd})$ as described below:

$$
\mathrm{P}_{\mathrm{P}}\left(O_{i}\right)=\left(\frac{k_{1}}{k_{1}+f\left(O_{i}\right)}\right)^{2}
$$

(1)

$\left.\begin{array}{lll}2 f\left(O_{i}\right) & \text { IF } & f\left(O_{i}\right) \\ \text { - } & \mathrm{P}_{\mathrm{d}}\left(O_{i}\right)=\end{array}\right\}<k_{2} \mathrm{z}$

(2)
Gutowitz [13] improved this model by giving the ants the capacity to sense the complexity of their neighborhood. The ants would not try to pick or drop anything in areas with low complexity. These complexity-seeking ants were able to avoid actions that did not contribute to the clustering process, performing their task more efficiently. Monmarche [9] proposed an algorithm where several objects are allowed to be on the same cell of the workspace grid. Each cell with one or more objects together corresponds to a cluster. Each ant is also capable of carrying more than one object at a time. Ngenkaew [14] proposed two multiple pheromone concepts in ant based clustering with ant nest algorithm and with ant memory algorithm. Artificial Trailing pheromone and Foraging pheromone help ants to decide which direction to go or where to 
pick up or drop the item of food. Handl [15] presented a comparative study of the performance of ant-based clustering against traditional methods. Study realized that ant clustering is very fast algorithm for high-dimensionality data and its interesting features like no assumption on the shape of the clusters, automatic identification of the number of the clusters and robust behavior to the effects of outliers.

Brown et. al [16] proposed hierarchical clustering by making a movement zone around each cluster. Worker ants move only in this zone. Then the resulting clusters are merged by the queen ant through a connection. Li et. al [17] proposed a new for calculating the distance based on reachability paths between two objects, called distance with connection. Jiang et. al [18] redefined the behavior of ant and colony similarity. Groups of objects are activated one by one to be artificial ants. This method does not search for the sample objects as ants are the objects themselves. It can reduce the number of iterations. Feghi et. al [19] presented a new Adaptive Ant-based Clustering Algorithm for clustering data sets. The algorithm takes into account the properties of aggregation pheromone and perception of the environment.

\section{B. ANTClass algorithm}

Monmarche [20] [21] combined the stochastic and exploratory principles of clustering ants with the deterministic and heuristic of the popular k-means algorithm in order to improve the convergence of the ant-based clustering algorithm. The proposed hybrid method is called AntClass and is based on the work of Lumer and Faieta [11]. The AntClass algorithm allows an ant to drop more than one object in the same cell, forming heaps of objects. Another important contribution of this algorithm is that it also makes use of hierarchical clustering, implemented by allowing ants to carry an entire heap of objects

\section{ALARMS FILTERING SYSTEM BASED ON ANTCLASS ALGORITHM}

This section describes the structure and the implementation of the alarms filtering system. This system try to adapt ANTClass algorithm to alarms filtering problem it consists of 3 modules: alarms preprocessing, ANT algorithm application, K-means algorithm Application. In fig 1 we present the general architecture of the proposed system.

Before detailing our approach we suggest presenting the notion of typical behavior which constitutes the central point of this approach. We believe that the various types of alarms generated by one NIDS for every couple of machines in connection in an interval of time can be representative of the nature of this session. Besides, this behavior can be similar for several machines in connection for the different periods. Then, the classification of this similar behavior in a number of typical behaviors can create coherent clusters of the data which can be significant potential scenarios of attacks.

\section{A. Alarms preprocessing module}

Firstly this module receives alarms from different NIDS installed in the network and extracts important information from each brut alert received. Alerts are retrieved from log files generated by NIDS and they represent an events produced by many external machines (with IPextern) trying to connect to a number of internal machines (with IPintern). Alarms preprocessing module creates a number of data vector called VC from alerts wich reflect intruder behavior in window of time. For a certain window of time, the numbers of alarms of type $i$ for each pair (IPint, IPext) is calculated. As a result, an aggregated alarm data vector called VC is represented below:

[DT, IPextern, IPintern] = \#a1, \#a2. . \#an where DT represents an interval of time,

IPextern represents the IP of external machines 
International Journal of Advanced Research in Computer and Communication Engineering Vol. 3, Issue 12, December 2014

IPintern represents the IP of internal randomly on the $2 \mathrm{D}$ board. Then each ant performs machines,

a move and possibly drops or pick up an object \#ai represents the number of alarms of type (which represent in our case a data vector). Each i. ant selects a random direction (among the 8 possible directions). Then each ant has a probability In the next sections we propose to exploit results of Pdc to further continue in this direction when moving alarms preprocessing module in order to create a next, else it flows randomly a new direction. We classification of intruders' behaviors using ant note that each ant has a speed parameter which clustering method called ANTClass which a tells of how many steps it will move in the selected hybridization of Ant clustering and K-means algorithm. direction before stopping again also the ant has a
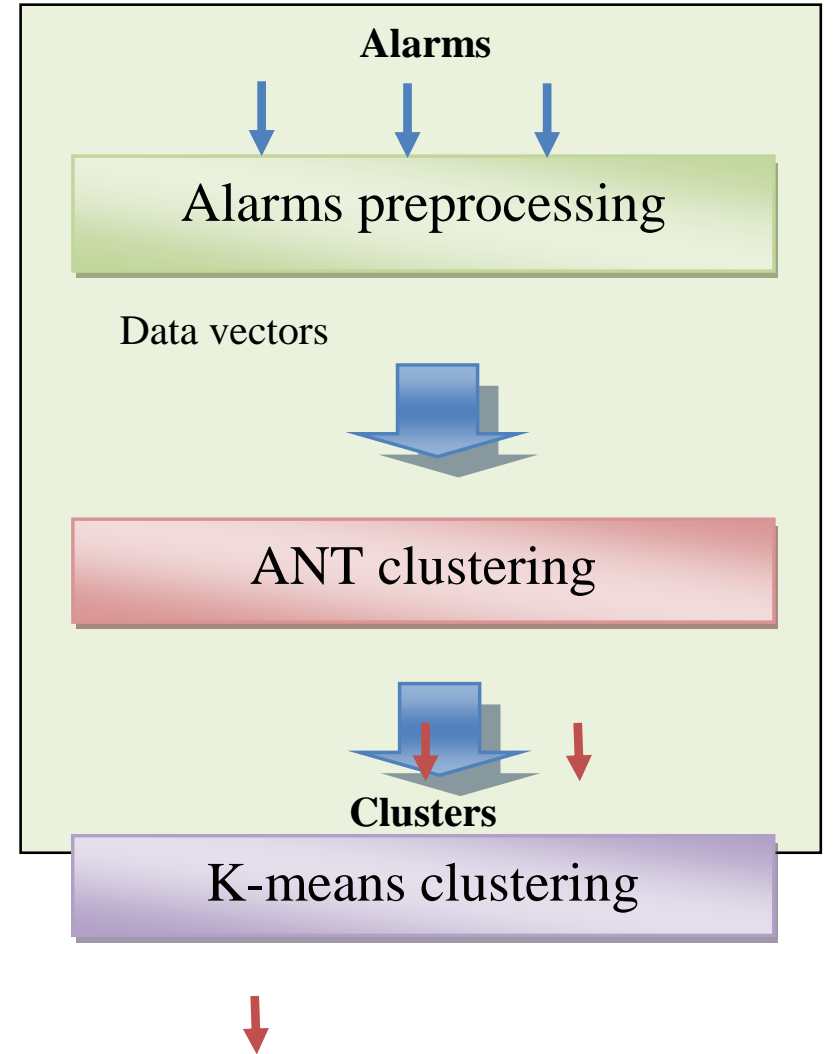

Fig1. Alarm filtering system architecture

\section{B. Application of ANT algorithm}

This module receives data vectors produced by an object has been assigned to a wrong heap then "alarms preprocessing module" in order to create a it can take a long time until the object is preliminary classification of intruders' behaviors which transported to the right cluster. So we propose to reflect the different actions of intruders in local combine ant based clustering algorithm with k-means machine. Hence, we use the Ant algorithm to apply algorithm in order deal with those problems. Hence, clustering of data vectors; the ant algorithm is in this section we use k-means algorithm to remove presented in fig 2 . Firstly the ants are located 
International Journal of Advanced Research in Computer and Communication Engineering Vol. 3, Issue 12, December 2014

quickly obvious errors and provide an efficient attack against apache server, 3 scenarios brute force heuristic for assigning "isolated" objects. against FTP server, and 1 scenario SNMP attack[1]. The Ant algorithm provide a initial partition of clusters, then K-means algorithm computes the center of each clusters then it computes a new partition by assigning every object to the heap with center is the closest to that object. This cycle is repeated during a given number of iteration or until the assignment has not changed during one cycle. In fig 3 we present K-means algorithm. The

1. Take as input the partition $P$ of the data set found by the ants in the form of $k$ heaps $H 1, \ldots, H k$,

2. Repeat

(a) Compute $\mathrm{O}$ center $(\mathrm{H} 1), \ldots, \mathrm{O}$ center $(\mathrm{Hk})$,

(b) Remove all objects from all heaps,

(c) For each object $\mathrm{Oi} \in \mathrm{E}$ :

i. Let $\mathrm{Hj}, \mathrm{j} \in[1, \mathrm{~K}]$ be the heap which center is the closest to Oi,

ii Accian $\mathrm{Oi}$ to $\mathrm{Hi}$ In order to evaluate our system, we have divided the dataset into 4 sets as it shown in Table 1.

Table 1.Test and training data set

\begin{tabular}{|c|c|c|c|}
\hline Data set & $\begin{array}{c}\text { Number } \\
\text { of alerts }\end{array}$ & $\begin{array}{c}\text { Number of } \\
\text { data vectors }\end{array}$ & $\begin{array}{c}\text { Number of } \\
\text { windows }\end{array}$ \\
\hline Test 1 & 166 & 50 & 10 \\
\hline Test 2 & 2815 & 1693 & 159 \\
\hline Test 3 & 11962 & 4000 & 330 \\
\hline Training & 32031 & 12000 & 435 \\
\hline
\end{tabular}

\section{B. Experimentation}

The main criteria that we have considered in the experimentation of our system are the detection rate, false positive rate and false negative rate.

- Detection Rate (DR): is defined as the number of attack scenario correctly classified by our system divided by the total number of test examples.

- $\quad$ False positive rate (FPR) : is defined as the proportion that normal data is falsely detected as attack behavior

Fig 3.K-means algorithm

False negative rate (FNR): is defined as the

\section{A. Implementation}

We have implemented our proposed approach with java language, using eclipse software, and we have applied this approach to a real dataset which retrieved from NIDS: Snort [1] log files. These files included 32031 alarm event records generated over duration of 20 days, from 20/11/2004 to 10/12/2004. These alarm events were produced by 4638 external machines trying to connect to 288 internal machines. The logs generated by SNORT include 16 real attack scenarios and one non-attack/normal scenario as identified by the domain expert. Attack scenarios include: 4 scenarios brute force on POP3, 3 scenarios crawler Web, 2 scenarios Web IIS, 2 scenarios scanner of vulnerability, 1 scenario IIS proportion that attack data is falsely detected as normal behavior

Table 2.Comparison of results

\begin{tabular}{|c|c|c|c|}
\hline Data set & FPR & FNR & DR \\
\hline Test 1 & 166 & 50 & 10 \\
\hline Test 2 & 2815 & 1693 & 159 \\
\hline Test 3 & 11962 & 4000 & 330 \\
\hline Training & 32031 & 12000 & 435 \\
\hline
\end{tabular}

Table 2 shows that the low rate of FP and FN are generated with Test3 data set, so we can note that the rate was improved by increasing the size of the dataset; what means that the addition of new normal connections moves closer to the profile establishes in the real profile of the users. Also the 
Table 2 shows that DR is improved by increasing the number of data set element.

Table 3.Comparison of results

\begin{tabular}{|c|c|c|}
\hline Approach & FPR & FNR \\
\hline ANTClass & $2 \%$ & $2 \%$ \\
\hline GHSOM & $4.7 \%$ & $4 \%$ \\
\hline SOM & $15 \%$ & $16 \%$ \\
\hline
\end{tabular}

Table 3 shows the results of our proposed ANTClass on the testing data and how it compares with other system which used the same datasets: GHSOM [1] and SOM [2]. Results show high performance of our system. The approach based on ANTClass gives the smallest false alerts rates. ANTClass outperforms SOM and GHSOM on false positives and false negatives. Our system showed a reduction in false positives to $2 \%$ and of false negatives to $2 \%$.

\section{CONCLUSION}

We have proposed a data mining technique based on ANTClass algorithm, to deal with the problem of the huge number false alarms, which represent a new decision support layer for network administrators to analyze and sort out alarms generated by a network intrusion detection system (NIDS). The application of our system in alarms filtering in intrusion detection context helps detect attacks and reduce false alarms with very considerable rates. There are still some challenges in our system. First, we can improve the clustering method in order to approximate the right number of classes. Secondly we can improve our system by integrating a prediction module which allows the prevention of intruders' actions.

\section{ACKNOWLEDGMENT}

The authors would like to thank Dr. Ahmed Faour of Beirut Arab University, Lebanon. for his comments on an initial version of this work.

\section{REFERENCES}


Biomedical Engineering and Informatics, IEEE 978-1-4244-

6498-2/10, pp. 2368-2372, 2010.

[19] I. El-Feghi, M. Errateeb, M. Ahmadi and M. A. Sid-Ahmed, 'An Adaptive Ant-Based Clustering Algorithm with Improved Environment Perception' International Conference on Systems, Man, and Cybernetics, San Antonio, TX, USA - October 2009 published in IEEE 978-1-4244-2794-9/09, pp. 1431-1438, 2009.

[20] N. Monmarche, M. Slimane and G. Venturini, "On improving clustering in numerical databases with artificial ants", Advances in Artificial Life, 1999, pp. 626-635.

[21] N. Monmarche, "On Data Clustering with Artificial Ants", In: Freitas AA, (ed.), Data Mining with Evolutionary Algorithms: Research Directions - Papers from the AAAI Workshop, AAAI Press, 1999, pp. 23-26. 